\title{
Orosomucoid in urine predicts cardiovascular and over-all mortality in patients with Type II diabetes
}

\author{
M. S. Christiansen ${ }^{1}$, E.Hommel ${ }^{2}$, E.Magid ${ }^{1}$, B.Feldt-Rasmussen ${ }^{3}$ \\ ${ }^{1}$ Department of Clinical Biochemistry, Amager Hospital, Copenhagen S, Denmark \\ ${ }^{2}$ Department of Internal Medicine, Bispebjerg Hospital, Copenhagen, Denmark \\ ${ }^{3}$ Department of Nephrology and Endocrinology, Rigshospitalet, Copenhagen, Denmark
}

\section{Abstract}

Aims/hypothesis. Urinary orosomucoid excretion rate is increased in a substantial proportion of patients with Type II (non-insulin-dependent) diabetes mellitus and normal urinary albumin excretion rate. The aim of this study was to determine whether increased urinary orosomucoid excretion rate is predictive of increased mortality in patients with Type II diabetes.

Methods. In a cohort study including 430 patients with Type II diabetes, baseline urinary samples were analysed for orosomucoid and albumin. Mean follow-up was 2.4 years.

Results. We found that 188 (44\%) patients had normal and $242(56 \%)$ patients had increased urinary orosomucoid excretion rates. During the study period 41 patients died; out of these 23 patients died of cardiovascular diseases. Odds ratio for all-cause mortality was 2.50 (95\% CI 1.00-6.22) and odds ratio for cardiovascular mortality was 9.81 (1.31-73.6) having increased urinary orosomucoid excretion rate at baseline (odds ratios adjusted for age, sex, duration of dia- betes, cardiovascular diseases, weight, medication, $\mathrm{HbA}_{1 \mathrm{c}}$, plasma creatinine and urinary albumin excretion rate).

Urinary albumin excretion rate was an independent predictor of all-cause mortality when urinary orosomucoid excretion rate was not included in the analysis. Subgroup analysis revealed that $39 \%$ of the patients with normal urinary albumin excretion rate $(n=251)$ had increased urinary orosomucoid excretion rates and that these patients had a higher cardiovascular mortality $(p=0.007)$ than patients with normal urinary albumin excretion rate and normal urinary orosomucoid excretion rates.

Conclusion/interpretation. We found that urinary orosomucoid excretion rate predicted all-cause and cardiovascular mortality in patients with Type II diabetes independently from other risk factors. [Diabetologia (2002) 45: 115-120]

Keywords Urinary orosomucoid, Type II diabetes, microalbuminuria, cardiovascular mortality, all-cause mortality.
Patients with Type II (non-insulin-dependent) diabetes mellitus have an increased risk of cardiovascular and all-cause mortality compared to the background

Received: 24 July 2001 and in revised form: 17 September 2001

Corresponding author: M. S. Christiansen, MD, Department of Clinical Biochemistry, Amager Hospital, Italiensvej 1, DK2300 Copenhagen S, Denmark (e-mail: merete.skovdal@ah. hosp.dk)

Abbreviations: UOER, Urinary orosomucoid excretion rate; UAER, urinary albumin excretion rate. population $[1,2]$. Microalbuminuria is a well-known predictor of increased mortality in patients with Type II diabetes [2-6]. The excess mortality is mainly caused by cardiovascular diseases $[1,2,6]$.

Orosomucoid is a glycoprotein $\left(41000 \mathrm{M}_{\mathrm{r}}\right.$, content of carbohydrate $42 \%$ ) with a low isoelectric point of 2.7 [7]. Orosomucoid is synthesized in the liver and its serum concentrations are raised in conditions of inflammation and tissue repair [8]. The specific function of the protein is not known. In renal physiological investigations orosomucoid has been classified as a marker of increased glomerular permeability [9]. 
In studies on healthy persons, urinary orosomucoid excretion rate (UOER) is very low [9-11]. It has been shown that UOER increases in parallel with urinary albumin excretion rate (UAER) in physiological proteinuria [9] and in patients with acute cardiovascular diseases [12]. In a study of 171 patients with Type II diabetes it was found that a substantial proportion of patients with normal UAER had increased UOER [11]. In the same study urinary orosomucoid increased in parallel with albumin in patients with micro-albuminuria and macroalbuminuria.

We speculate that increased UOER in patients with Type II diabetes might be an earlier predictor than microalbuminuria of cardiovascular mortality and morbidity. The aim of our study was to determine whether increased UOER is predictive of all-cause and cardiovascular mortality in patients with Type II diabetes.

\section{Subjects and methods}

Subjects. A cohort study consecutively including all patients with Type II diabetes and dipstick-negative over-night urine samples attending the outpatient clinic at Amager Hospital, Copenhagen was carried out from September 1995 to December 1998. A total of 430 patients were followed until death, emigration or until 31 December 1999.

Medical history was obtained retrospectively from patient records. The diagnosis of Type II diabetes was based on the World Health Organization criteria of 1985 (fasting blood glucose $\geq 7.8 \mathrm{mmol} / \mathrm{l}$ or 2-h OGTT values of blood glucose $\geq 11.1 \mathrm{mmol} / \mathrm{l}$ and not being prone for keto-acidosis). Duration of diabetes was defined as duration from the date of diagnosis. Hypertension was defined as either having BP at rest of more than 140/90 $\mathrm{mmHg}$ at several measurements with mercury manometer or by a history of hypertension and anti-hypertensive medication. Coronary heart disease was defined as a history of angina pectoris, previous myocardial infarction, presence of ST-segment depression of $2 \mathrm{~mm}$, or more, on electrocardiography or during an exercise test or, when available, positive coronary arteriography. Previous myocardial infarction was diagnosed according to the World Health Organization criteria as two out of three of the following findings: a history of chest pain, an ST-segment elevation on electrocardiography and/or an increase of biochemical parameters in blood over time. Stroke was registered in patients with a history of neurologic deficit lasting more than $24 \mathrm{~h}$ and, when available, visualization on computed tomography as haemorrhages or ischemic infarction. Congestive heart failure was defined as a history of dyspnoea and peripheral edema, positive physical examination with pulmonary congestion and positive chest $\mathrm{x}$-ray examination and/or echocardiography when available. Ophthalmologists using fundoscopy through dilated pupils diagnosed diabetic retinopathy. Simple retinopathy was diagnosed in the presence of changing calibre of retinal blood vessels, microaneurysms or tiny exudates. Proliferative retinopathy was diagnosed in the presence of new vessel formation, exudates, scarring or haemorrhage. Patients who never smoked were registered as non-smokers. Patients smoking were registered as smokers. Patients who stopped smoking were registered as former smokers. Medical treatment was registered as treatment with insulin or none, and treatment with ACE-in- hibitors or none. Information about causes of death was gathered through the central Danish registry of causes of death and through death certificates from journal files and registered according to the International Classification of Diseases (ICD 10).

The local committee of ethics at Copenhagen approved the study protocol and the study was carried out in accordance with the Helsinki Declaration II.

Urine analysis. At baseline all patients collected timed overnight urine samples, which were stored without additives at $5^{\circ} \mathrm{C}$ and analysed within a week from the collection time. All samples were analysed for leucocytes, nitrite or haemoglobin by Multistix (Bayer, Elkhart, Ind. USA) at the day of delivery of the urine sample to the laboratory. Patients with urine samples positive for leucocytes, nitrite or haemoglobin were excluded from the study $(n=104)$. Patients with missing values of time of urine collection $(n=22)$, patients with urine collection time over $12 \mathrm{~h}(n=15)$ and patients with urine creatinine concentrations of less than $1.5 \mathrm{mmol} / \mathrm{l}(n=6)$ were excluded from the study.

Urinary orosomucoid and albumin were analysed with an in-house method by immunoturbidimetry (Cobas Mira, Roche, Basel, Switzerland) with reagents from Roche and DAKO (Copenhagen, Denmark) and calibrated using human serum protein calibrator X 908 DAKO.

Excretion rates were calculated for orosomucoid and albumin by the formula: (urine protein concentration $\mathrm{x}$ urine volume)/urine collection time. Detection limits and intra-series imprecision (CV\%) were: urinary orosomucoid $1.2 \mathrm{mg} / \mathrm{l}$ $(5.6 \%)$ and urinary albumin $3.3 \mathrm{mg} / \mathrm{l}(3.0 \%)$.

We did not find published reference data for turbidimetric analysis of urinary orosomucoid. We have made a pilot study (data not shown) of 19 healthy persons, where we found that all but two persons had urinary orosomucoid concentrations below the detection limit. To achieve a high sensitivity of the analysis we chose a value just above the detection limit as a cut-of value for UOER. This value is comparable to the $95^{\text {th }}$ percentile of UOER in the control group in reference [11] in which orosomucoid was measured by means of a radioimmunoassay.

Cut-off values were UOER greater than $0.88 \mu \mathrm{g} / \mathrm{min}$ [11] and UAER greater than $20 \mu \mathrm{g} / \mathrm{min}$ [13]. Microalbuminuria was defined as a UAER of 20 to $200 \mu \mathrm{g} / \mathrm{min}$ [13]. Macroalbuminuria was defined as a UAER greater than $200 \mu \mathrm{g} / \mathrm{min}$.

Blood analysis. Information about blood $\mathrm{HbA}_{1 \mathrm{c}}$ and plasma creatinine was collected from routine blood analyses carried out within a maximum period of 6 months before and after the corresponding urine sample. Blood $\mathrm{HbA}_{1 \mathrm{c}}$ was analysed on DCA 2000 (Bayer). Plasma and urinary creatinine were analysed by an enzymatic method on Vitros 250 (Johnson and Johnson, Rochester, N. Y., USA) until June 1998 and subsequently by a modified Jaffé reaction (Cobas Integra, Roche, Basel, Switzerland).

Statistics. Statistica for Windows version 5.1 (StatSoft, Tulsa, Okla., USA) was used for statistical analyses. A chi-square test was used for dichotomous variables and a Mann-Whitney $\mathrm{U}$ test was used for continuous variables. Nonparametric correlations were done using Spearman's rank order correlation. Many patients had urinary orosomucoid concentrations below the detection limit. Therefore we had to make this parameter a dichotomous variable in multivariate analysis. The median values and correlations were calculated for patients with increased values of UOER as a continuous variable. Survival analysis was done by the method of Kaplan-Meier and survival 
Table 1. Demographic and clinical data at baseline for patients with normal vs increased urinary orosomucoid excretion rates (UOER)

\begin{tabular}{|c|c|c|c|}
\hline & Normal UOER $n=188$ & Increased UOER $n=242$ & $p$ value \\
\hline Sex (men/women) & $97 / 91$ & $176 / 66$ & $<0.001^{\mathrm{a}}$ \\
\hline Age $(\text { years })^{\mathrm{b}}$ & $57(11)$ & $60(11)$ & $<0.003^{\mathrm{c}}$ \\
\hline Duration of diabetes (years) ${ }^{\mathrm{d}}$ & $3(0.02-30)$ & $4(0.02-37)$ & $<0.04^{\mathrm{c}}$ \\
\hline Plasma creatinine $(\mu \mathrm{mol} / \mathrm{l})^{\mathrm{b}}$ & $71(20)^{f}$ & $85(32)^{\mathrm{g}}$ & $<0.001^{\mathrm{c}}$ \\
\hline UAER $(\mu \mathrm{g} / \mathrm{min})^{\mathrm{d}}$ & $11(2.7-421)$ & $29(2.7-3436)$ & $<0.001^{\mathrm{c}}$ \\
\hline Systolic BP $(\mathrm{mmHg})^{\mathrm{b}}$ & $144(23)^{\mathrm{h}}$ & $148(23)^{\mathrm{g}}$ & $0.06^{\mathrm{c}}$ \\
\hline Hypertension, $n(\%)$ & $70(37)$ & $130(54)$ & $<0.001^{\mathrm{a}}$ \\
\hline Heart failure, $n(\%)$ & $10(5)$ & $39(16)$ & $<0.001^{\mathrm{a}}$ \\
\hline Coronary heart disease, $n(\%)$ & $36(19)$ & $52(21)$ & $0.63^{\mathrm{a}}$ \\
\hline Previous myocardial infarction, $n(\%)$ & $23(12)$ & $34(14)$ & $0.68^{\mathrm{a}}$ \\
\hline Stroke, $n(\%)$ & $9(5)$ & $20(8)$ & $0.22^{\mathrm{a}}$ \\
\hline Retinopathy, simplex/proliferative (\%) & $23 / 1(14 / 1)^{\mathrm{k}}$ & $40 / 8(19 / 4)^{1}$ & $<0.05^{\mathrm{e}}$ \\
\hline Smoking, $n$ (no/yes/former) & $89 / 72 / 5^{\mathrm{m}}$ & $117 / 101 / 4^{\mathrm{n}}$ & $0.70^{\mathrm{e}}$ \\
\hline
\end{tabular}

UAER, urinary albumin excretion rate

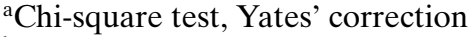

${ }^{\mathrm{b}}$ Means (SD)

'Mann-Whitney U test

curves were compared by a log-rank test. Cox proportional hazards regression analysis with backward stepwise regression was used for multivariate analysis. Subjects with missing values were excluded from the multivariate analysis. Log-transformed values of UAER were used in the multivariate analysis. A $p$ value of less than 0.05 was considered statistically significant.

\section{Results}

We studied 430 patients with a mean age of 59 years (SD 11) for a mean period of 2.4 years (range $0.01-4.2)$. Patients were sub-classified in relation to normal or increased UOER at baseline (Table 1). There were $188(44 \%)$ patients with normal UOER and $242(56 \%)$ with increased UOER. Patients with increased UOER had median UOER values of $10.7 \mu \mathrm{g} / \mathrm{min}$ (inter quartile range 5.7-22.0).

There were $251(58 \%)$ patients with normal UAER and 179 (42\%) with increased UAER. Patients with microalbuminuria totalled $130(30 \%)$ and $49(11 \%)$ had macroalbuminuria. Patients with increased UOER had a parallel increase in UAER $(r=0.70, p<0.001 ; n=242)$.

Increased UOER was correlated to plasma creatinine $(r=0.30, p<0.001 ; n=237)$ and increased UOER was weakly correlated to duration of diabetes $(r=0.19, \quad p<0.004 ; \quad n=241), \quad$ weight $\quad(r=0.13$, $p<0.05 ; n=231)$ and systolic BP $(r=0.13, p<0.05$; $n=237)$. Increased UOER was not correlated to age, blood $\mathrm{HbA}_{1 \mathrm{c}}$ or diastolic BP.

In total, 41 patients died and one patient emigrated. No patients were lost to follow-up. Causes of

\begin{abstract}
${ }^{\mathrm{d}}$ Median (range)
ePearson Chi-square

$n$ (corrected for missing values $)={ }^{\mathrm{f}} 183,{ }^{\mathrm{g}} 238,{ }^{\mathrm{h}} 186,{ }^{\mathrm{i}} 182,{ }^{\mathrm{j}} 232$, ${ }^{\mathrm{k}} 161,{ }^{1} 207,{ }^{\mathrm{m}} 166,{ }^{\mathrm{n}} 222$
\end{abstract}

death were: cardiovascular diseases $(n=23)$, neoplasm's $(n=6)$, infections $(n=4)$, unknown $(n=3)$, uraemia $(n=2)$, Type II diabetes $(n=2)$, suicide $(n=1)$. Seven $(4 \%$ of 188$)$ patients with normal UOER died compared with 34 (14\% of 242) patients with increased UOER $(p<0.001)$. The Kaplan-Meier curves for overall survival are shown (Fig. 1).

A total of 23 patients died of cardiovascular diseases: eight patients died of heart failure, eight died of myocardial infarction, four died of stroke and three died suddenly. Out of the patients who died of cardiovascular diseases two patients had normal UOER and 21 patients had increased UOER at baseline $(p<0.001)$.

Multivariate analysis including all baseline variables (except retinopathy and smoking) showed that higher age, plasma creatinine, a history of stroke, increased UOER and lower weight at baseline, were independently associated with increased all-cause mortality. Higher age, plasma creatinine, history of coronary heart disease and increased UOER were independently associated with increased cardiovascular mortality. The variables classifying degree of retinopathy $(n=368)$ and smoking habits $(n=388)$ were not included in the multivariate analysis because of a high number of patients with missing values. Patients with increased UOER had a 2.50 (95\% CI 1.00-6.22; $p<0.05$ ) greater risk of all-cause mortality (Table 2) and a $9.81(1.31-73.6 ; p<0.03)$ greater risk of cardiovascular mortality than patients with normal UOER (Table 3).

Further sub-classification of the patients with normal UAER $(n=251)$ showed $98(39 \%)$ patients 


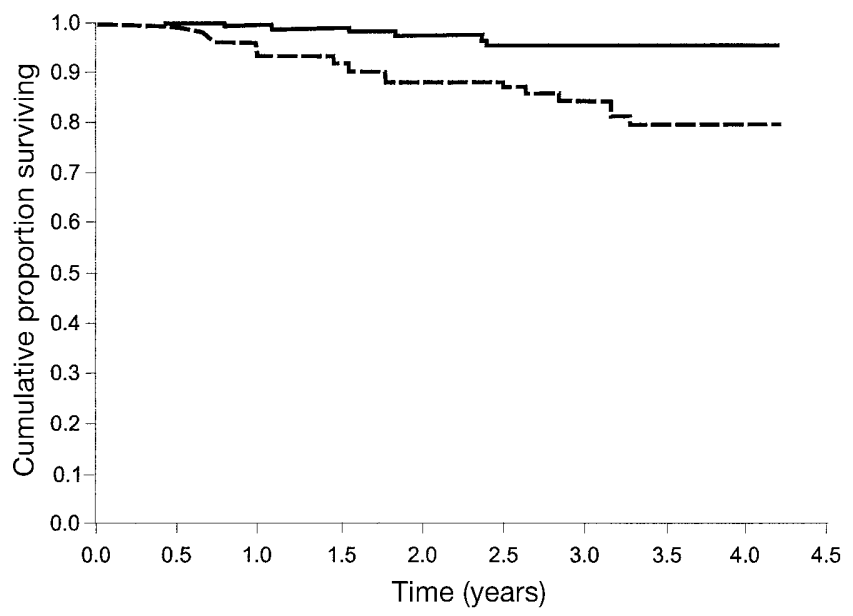

Fig.1. Kaplan-Meier survival curves for patients with normal (-) vs increased (---) urinary orosomucoid excretion rates at baseline; $p<0.001$ (log-rank test)

with increased UOER [median UOER values: $5.9 \mu \mathrm{g} /$ min (inter quartile range 3.4-9.2)]. More men than women $(p<0.03)$ showed increased UOER and normal UAER and had higher blood $\mathrm{HbA}_{1 \mathrm{c}}(p<0.02)$ and plasma creatinine $(p<0.001)$ than patients with normal UOER and UAER. At baseline no differences between the two subgroups were found in prevalence of cardiovascular diseases or risk factors for cardiovascular diseases except sex.

Fourteen patients with normal UAER died. Of these patients six had normal UOER and eight had increased UOER at baseline (all-cause mortality; log-rank test: $p=0.15)$. Out of these ten patients died of cardiovascular diseases (two patients with normal UOER and eight patients with increased UOER; cardiovascular mortality: $p=0.007$ ). Because of a small number of deaths in the subgroups, multivariate analysis could not be carried out. A subgroup of patients $(n=34)$ had increased UAER and normal UOER. None of these patients died.

Of 14 patients with normal UAER $14(n=251)$ died compared with 27 patients with increased UAER $(n=179 ; p<0.001)$. Ten patients with normal UAER and 13 patients with increased UAER died of cardiovascular diseases $(p=0.10)$. When UOER was not included in the multivariate analysis the logarithm to UAER predicted all-cause mortality (odds ratio was $1.26,95 \%$ CI $1.01-1.56, p<0.04)$ along with age $(p<0.001)$, plasma creatinine $(p<0.001)$, a history of stroke $(p<0.007)$ and lower weight $p<0.04)$ at baseline. The logarithm to UAER did not independently predict cardiovascular mortality, when UOER was not included in the multivariate analysis.
Table 2. Multiple regression analysis in relation to over-all mortality

\begin{tabular}{lll}
\hline Variable & $\begin{array}{l}\text { Hazard ratio } \\
(95 \% \mathrm{CI})\end{array}$ & $p$ value \\
\hline Age (years) & $1.09(1.04-1.13)$ & $<0.001$ \\
Plasma creatinine (per 10 $\mathrm{mol} / \mathrm{l})$ & $1.25(1.15-1.35)$ & $<0.001$ \\
Stroke (no/yes) & $3.06(1.24-7.57)$ & $<0.02$ \\
UOER (normal/increased) & $2.50(1.00-6.22)$ & $<0.05$ \\
Weight (kg) & $0.98(0.95-1.00)$ & $<0.05$ \\
\hline
\end{tabular}

Data are for selected patients $(n=402$, subjects with missing values are excluded)

Table 3. Multiple regression analysis in relation to cardiovascular mortality

\begin{tabular}{lll}
\hline Variable & $\begin{array}{l}\text { Hazard ratio } \\
(95 \% \mathrm{CI})\end{array}$ & $p$ value \\
\hline Age (years) & $1.10(1.04-1.17)$ & $<0.001$ \\
Plasma creatinine (per 10 $\mu \mathrm{mol} / \mathrm{l})$ & $1.18(1.04-1.33)$ & $<0.008$ \\
Coronary heart disease (no/yes) & $4.35(1.70-11.09)$ & $<0.003$ \\
UOER (normal/increased) & $9.81(1.31-73.6)$ & $<0.03$ \\
\hline
\end{tabular}

Data are for selected patients $(n=402$, subjects with missing values are excluded)

\section{Discussion}

This study shows that UOER is an independent predictor of increased all-cause and cardiovascular mortality in patients with Type II diabetes.

Compared to patients with normal UOER, patients with increased UOER had a preponderance of hypertension, heart failure, longer duration of diabetes, were older, mostly men and had higher values of $\mathrm{HbA}_{1 \mathrm{c}}$, plasma creatinine and UAER. All these variables, however, were adjusted for in the multivariate analysis showing UOER as an independent predictor of increased cardiovascular and all-cause mortality.

Patients with Type II diabetes and increasing values of UAER have been found to have a higher prevalence of arterial hypertension, proliferative retinopathy, longer duration of diabetes and higher $\mathrm{HbA}_{1 \mathrm{c}}$ values than patients with normal UAER [14]. In our study mean values of age, duration of diabetes, $\mathrm{HbA}_{1 \mathrm{c}}$, plasma creatinine and prevalence of microalbuminuria and macroalbuminuria at baseline were comparable to studies investigating the relation of UAER and mortality [2-6]. We therefore assume that our cohort of patients is a representative group of patients with Type II diabetes.

We confirmed the previous observation that patients with Type II diabetes could be sub-classified using UOER as a marker [11]. In the subgroup analysis of patients with normal UAER, 39\% had increased UOER and this combination was associated with increased cardiovascular mortality compared with patients with normal UOER and normal UAER. This indicates that UOER could be a valuable and very 
early risk marker of cardiovascular disease in patients with Type II diabetes and normal UAER.

Although the follow-up time (2.4 years) was very short, we found UOER to be an independent predictor of cardiovascular and overall mortality. Urinary albumin excretion rate is a well-known predictor of cardiovascular morbidity and mortality in patients with Type I diabetes [15-17], Type II diabetes [2-6] and in non-diabetic persons [18-20] as well. In our study, however, UAER predicted all-cause mortality only when UOER was not included in the analysis and UAER was not an independent predictor of cardiovascular mortality. The short follow-up period in our study compared to a longer follow-up time of 5 to 13 years in other studies [2-6] might explain this discrepancy.

Increased UAER was not always followed by an increase in UOER. In this subgroup no patients died in the follow-up period. We found that lower weight predicted all-cause mortality. A possible interpretation could be a lower weight at baseline of high-risk patients since the study population in general is adipose.

The pathophysiological background for our findings of an association between UOER and mortality is not known. Normal plasma concentrations of orosomucoid are less than $2 \%$ of albumin $[9,21]$. Orosomucoid is a smaller molecule than albumin and probably because of this, the renal clearance of orosomucoid in normal persons is 5 to 10 times higher than albumin, depending on the urinary collection method $[9,21]$. This might explain UOER being a more sensitive marker than albumin for increased membrane permeability. Because of the high renal clearance, UOER is sensitive to changes in serum concentrations of orosomucoid and to changes in renal tubular function and renal blood flow.

As this study is partly retrospective we did not have access to serum values of orosomucoid. This is a weakness of the study and we are not able to distinguish between these possible explanations of our findings. However, increased serum-concentrations of orosomucoid have been shown independently to predict the development of Type II diabetes in middle-aged persons probably linking acute-phase response, cytokine actions and insulin sensitivity [22]. There might be a link between pro-inflammatory cytokine production, endothelial cell dysfunction and leakage of urinary proteins. Small, but increasing serum concentrations of orosomucoid in parallel with increasing serum concentrations of interleukin- 6 and urinary albumin excretion have been found in patients with Type II diabetes and even more pronounced in patients with coronary heart disease and increased risk factors of atherosclerosis [23].

We found that UOER independently of other risk factors predicted all-cause and cardiovascular mortality in patients with Type II diabetes in a short-term follow-up study. Even in a subgroup analysis of patients with normal UAER, increased UOER was strongly associated with cardiovascular mortality. We conclude that UOER complements UAER in patients with Type II diabetes and that UOER seems to be a valuable marker of increased risk of cardiovascular and all-cause mortality. Urinary orosomucoid excretion rate could be a more potent marker of cardiovascular mortality than microalbuminuria.

Acknowledgements. We are most grateful to S. H. Nielsen and L. Foged for their collaboration and technical support, to East Danish Research Forum on Health Sciences, Department of Biostatistics, University of Copenhagen for statistical support and to the Ernst and Vibeke Husman's Foundation, Copenhagen for financial support.

\section{References}

1. Morrish NJ, Stevens LK, Head J, Fuller JH, Jarrett RJ, Keen H (1990) A prospective study of mortality among middle-aged diabetic patients (the London Cohort of the WHO Multinational Study of Vascular Disease in Diabetics) I: causes and death rates. Diabetologia 33: 538-541

2. Gall M-A, Borch-Johnsen K, Hougaard P, Nielsen FS, Parving H-H (1995) Albuminuria and poor glycemic control predict mortality in NIDDM. Diabetes 44: 1303-1309

3. Jarrett RJ, Viberti GC, Argyropoulos A, Hill RD, Mahmud U, Murrells TJ (1984) Microalbuminuria predicts mortality in non-insulin-dependent diabetes. Diabet Med 1: $17-19$

4. Mogensen CE (1984) Microalbuminuria predicts clinical proteinuria and early mortality in maturity-onset diabetes. N Engl J Med 310: 356-360

5. Schmitz A, Vaeth M (1988) Microalbuminuria: A major risk factor in non-insulin-dependent diabetes. A 10-year follow-up study of 503 patients. Diabet Med 5: 126-134

6. Neil A, Hawkins M, Potok M, Thorogood M, Cohen D, Mann J (1993) A prospective population-based study of microalbuminuria as a predictor of mortality in NIDDM. Diabetes Care 16: 996-1003

7. Schmid K (1989) Human plasma $\alpha_{1}$-acid glycoprotein biochemical properties, the amino acid sequence and the structure of the carbohydrate moiety, variants and poly-

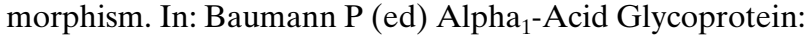
Genetics, Biochemistry, Physiological Functions, and Pharmacology. (Progress in clinical and biological research, Vol. 300) Liss, New York pp 7-22

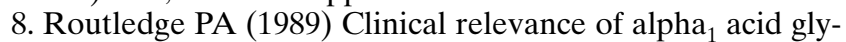
coprotein in health and disease. In: Baumann P (ed) Alpha $_{1}$-Acid Glycoprotein: Genetics, Biochemistry, Physiological Functions, and Pharmacology. (Progress in clinical and biological research, Vol. 300) Liss, New York pp 185-198

9. Poortmans JR, Haralambie G (1979) Biochemical changes in a $100 \mathrm{~km}$ run: Proteins in serum and urine. Eur J Appl Physiol Occup Physiol 40: 245-254

10. Vasson MP, Baguet JC, Arvieiller MR, Bargnoux PJ, Giroud JP, Raichvarg D (1993) Serum and urinary alpha-1 acid glycoprotein in chronic renal failure. Nephron 65:299-303

11. Ito S, Tsuda A, Momotsu T et al. (1989) Urinary orosomucoid excretion rate in patients with non-insulin-dependent diabetes mellitus. Acta Endocrinol (Copenh) 120: 584-590 
12. Nielsen SH, Petersen JS, Magid E (1999) Albuminuria in ischemic heart disease. Scand J Clin Lab Invest 59 [Suppl 230]: S143-S152

13. Mogensen CE, Keane WF, Bennett PH et al. (1995) Prevention of diabetic renal disease with special reference to microalbuminuria. Lancet 346: 1080-1084

14. Gall M-A, Rossing P, Skøtt P et al. (1991) Prevalence of micro- and macroalbuminuria, arterial hypertension, retinopathy and large vessel disease in European Type 2 (non-insulin-dependent) diabetic patients. Diabetologia 34: 655-661

15. Messent JWC, Elliott TG, Hill RD, Jarrett RJ, Keen H, Viberti GC (1992) Prognostic significance of microalbuminuria in insulin-dependent diabetes mellitus: A twenty-three year follow-up study. Kidney Int 41: 836-839

16. Deckert T, Yokoyama H, Mathiesen E et al. (1996) Cohort study of predictive value of urinary albumin excretion for atherosclerotic vascular disease in patients with insulin dependent diabetes. BMJ 312: 871-874

17. Rossing P, Hougaard P, Borch-Johnsen K, Parving H-H (1996) Predictors of mortality in insulin dependent diabetes: 10 year observational follow up study. BMJ 313: 779-784
18. Yudkin JS, Forrest RD, Jackson CA (1988) Microalbuminuria as predictor of vascular disease in non-diabetic subjects. Lancet ii: 530-533

19. Damsgaard EM, Frøland A, Jørgensen OD, Mogensen CE (1990) Microalbuminuria as predictor of increased mortality in elderly people. BMJ 300: 297-300

20. Borch-Johnsen K, Feldt-Rasmussen B, Strandgaard S, Schroll M, Jensen JS (1999) Urinary albumin excretion an independent predictor of ischemic heart disease. Arterioscler Thromb Vasc Biol 19: 1992-1997

21. Tencer J, Thysell H, Grubb A (1996) Analysis of proteinuria: reference limits for urine excretion of albumin, protein $\mathrm{HC}$, immunoglobulin $\mathrm{G}, \boldsymbol{x}$ - and $\lambda$-immunoreactivity, orosomucoid and $\alpha_{1}$-antitrypsin. Scand J Clin Lab Invest 56: 691-700

22. Schmidt MI, Duncan BB, Sharrett AR et al. (1999) Markers of inflammation and prediction of diabetes mellitus in adults (Atherosclerosis Risk in Communities study): a cohort study. Lancet 353: 1649-1652

23. Pickup JC, Mattock MB, Chusney GD, Burt D (1997) NIDDM as a disease of the innate immune system: association of acute-phase reactants and interleukin- 6 with metabolic syndrome X. Diabetologia 40: 1286-1292 